\title{
Periodontitis is related to lung volumes and airflow limitation: a cross-sectional study
}

\author{
Birte Holtfreter ${ }^{1}$, Stefanie Richter ${ }^{1}$, Thomas Kocher ${ }^{1}$, Marcus Dörr², \\ Henry Völzke ${ }^{3}$, Till Ittermann ${ }^{3}$, Anne Obst ${ }^{2}$, Christoph Schäper ${ }^{2}$, Ulrich John ${ }^{4}$, \\ Peter Meisel ${ }^{1}$, Anne Grotevendt ${ }^{5}$, Stephan B. Felix ${ }^{2}$, Ralf Ewert ${ }^{2}$ and Sven Gläser ${ }^{2}$
}

\begin{abstract}
Affiliations: 'Unit of Periodontology, Dept of Restorative Dentistry, Periodontology and Endodontology, University of Medicine, Ernst-Moritz-Arndt University Greifswald, Greifswald, ${ }^{2}$ Dept of Internal Medicine B Cardiology, Intensive Care, Pulmonary Medicine and Infectious Diseases, University of Medicine, Ernst-MoritzArndt University Greifswald, Greifswald, ${ }^{3}$ Institute for Community Medicine, Study of Health in Pomerania (SHIP)/Clinical-Epidemiological Research, University of Medicine, Ernst-Moritz-Arndt University Greifswald, Greifswald, 4 Institute of Epidemiology and Social Medicine, University of Medicine, Ernst-Moritz-Arndt University Greifswald, Greifswald, and ${ }^{5}$ nstitute of Clinical Chemistry and Laboratory Medicine, University of Medicine, Ernst-Moritz-Arndt University Greifswald, Greifswald, Germany.
\end{abstract}

Correspondence: B. Holtfreter, Ernst-Moritz-Arndt University Greifswald, University of Medicine, Dept of Restorative Dentistry, Periodontology and Endodontology, Unit of Periodontology, Rotgerberstr. 8, 17475 Greifswald, Germany. E-mail: birte.holtfreterवuni-greifswald.de

ABSTRACT This study aimed to assess the potential association of periodontal diseases with lung volumes and airflow limitation in a general adult population.

Based on a representative population sample of the Study of Health in Pomerania (SHIP), 1463 subjects aged 25-86 years were included. Periodontal status was assessed by clinical attachment loss (CAL), probing depth and number of missing teeth. Lung function was measured using spirometry, body plethysmography and diffusing capacity of the lung for carbon monoxide. Linear regression models using fractional polynomials were used to assess associations between periodontal disease and lung function. Fibrinogen and high-sensitivity C-reactive protein (hs-CRP) were evaluated as potential intermediate factors.

After full adjustment for potential confounders mean CAL was significantly associated with variables of mobile dynamic and static lung volumes, airflow limitation and hyperinflation $(\mathrm{p}<0.05)$. Including fibrinogen and hs-CRP did not change coefficients of mean CAL; associations remained statistically significant. Mean CAL was not associated with total lung capacity and diffusing capacity of the lung for carbon monoxide. Associations were confirmed for mean probing depth, extent measures of CAL/probing depth and number of missing teeth.

Periodontal disease was significantly associated with reduced lung volumes and airflow limitation in this general adult population sample. Systemic inflammation did not provide a mechanism linking both diseases.

@ERSpublications

Periodontal disease was significantly associated with reduced lung volumes and airflow limitation in an adult population http://ow.ly/oZcsc

This article has supplementary material available from www.erj.ersjournals.com

Received: July 162012 | Accepted after revision: Oct 162012 | First published online: Dec 062012

Conflict of interest: Disclosures can be found alongside the online version of this article at www.erj.ersjournals.com

Copyright @ERS 2013 


\section{Introduction}

Periodontitis is one of the most prevalent human infections. The majority of adults suffer from some degree of periodontitis, with $15-20 \%$ of the adult population having severe periodontal disease $[1,2]$. Periodontitis is an oral bacterial infection that results in gingival inflammation, breakdown of the supporting connective tissue, pocket formation between the gingiva and the tooth, destruction of alveolar bone and eventually exfoliation of teeth [3]. While probing depth reflects current disease status, clinical attachment loss (CAL) equals the reduction in the supporting connective tissue of the tooth and reflects cumulative disease experience.

To date, few studies have investigated the relationship between periodontitis [4-14] or oral hygiene [12, 13, 15] and respiratory diseases, although common causes might exist. Both diseases have been related to systemic inflammation [16-20]. Individuals with airflow limitation demonstrate airway and systemic inflammation, depending on the severity of lung disease, physical fitness and potential comorbidities [17-19]. Besides smoking [21] and obesity [22], risk factors for lung function decline and airflow limitation are ageing and pulmonary diseases, such as chronic obstructive pulmonary disease (COPD) [23].

Merely on the basis of a few spirometry parameters, some cross-sectional and case-control studies reported significant associations of periodontal disease with history of COPD and lung function decline [4-8, 12]. Furthermore, periodontal disease has been related to the longitudinal decline in spirometric lung volumes $[9,10,14]$. If periodontal disease causally contributed to a declining lung function as an independent risk factor, it would provide a rationale to consider periodontal prevention and treatment as therapeutic option to target a decreasing lung function, or at least to support dedicated treatments of pneumological diseases. However, the mechanisms behind these observations still remain unclear and controversial.

One theoretical pathway linking both diseases comprehends the aspiration of saliva harbouring oral bacteria into the lung [24]. Another pathway may be upregulated inflammation in the lungs by systemic low-grade inflammation induced by periodontal diseases [16]. Systemic inflammation markers have also been associated with reduced spirometric lung volumes [17-20]. So far, the interrelationship between periodontal disease, inflammation and lung function remains poorly understood.

Thus, the major aim of this study was to further clarify the association between periodontal disease and airflow limitation, lung volumes and diffusing capacity, based on a highly standardised setting of comprehensive lung function test parameters within the population-based Study of Health in Pomerania (SHIP). We further evaluated 1) the strength of confounding by smoking, height and educational status and 2) the role of systemic inflammation as an intermediate factor.

\section{Material and methods \\ Study population}

SHIP is a prospective cohort study in the northeast of Germany. The study was approved by the local institutional review board by the ethics committee. Details of the study design are given elsewhere $[25,26]$. Of 7008 randomly selected adults aged 20-79 years with German citizenship, 6262 were invited to participate. Of those, 4308 (68.8\%) individuals participated in the baseline study (SHIP-0, 1997-2001). Follow-up examinations (SHIP-1) were performed 5 years later, including 3300 subjects aged 25-85 years [27]. Of these, 1809 subjects ( 885 males and 924 females) participated in a substudy including body plethysmography, spirometry, helium dilution and diffusing capacity of the lung for carbon monoxide (TLCO) (fig. 1). The median (interquartile range) time interval between core examination of SHIP-1 and lung function examination was $18(0-74)$ days. We excluded those with missing lung function data $(\mathrm{n}=9)$, missing dental examinations $(n=8)$, regular intake of nonsteroidal antirheumatics or steroids $(n=274)$, high-sensitivity C-reactive protein (hs-CRP) $>10 \mathrm{mg} \cdot \mathrm{L}^{-1}(\mathrm{n}=42)$ indicating acute and active infection, systemic inflammatory processes or physical trauma [28], or missing confounder data $(n=13)$. For the remaining 1463 individuals with complete data, the number of missing teeth was available in all subjects, probing depth data were recorded in 1327 subjects and attachment loss data were available in 1268 subjects (fig. 1). In total, data from 1268 participants were evaluated for our main analyses.

Support statement: The Study of Health in Pomerania (SHIP) is part of the Community Medicine Research (CMR) Net of the University of Greifswald, Germany, which is funded by grants from the German Federal Ministry of Education and Research (BMBF; grants 01ZZ96030 and 01ZZ0701), the Ministry for Education, Research and Cultural Affairs and the Social Ministry of the Federal State of Mecklenburg-West Pomerania. The CMR encompasses several research projects that share data of the population-based SHIP (www.medizin.uni-greifswald.de). This work was supported by the Kompetenznetz Asthma/COPD (Competence Network Asthma/COPD) funded by the Federal Ministry of Education and Research (FKZ 01GI0881-0888). B. Holtfreter and S. Richter were supported by unlimited grants from GABA (Therwil, Switzerland). 


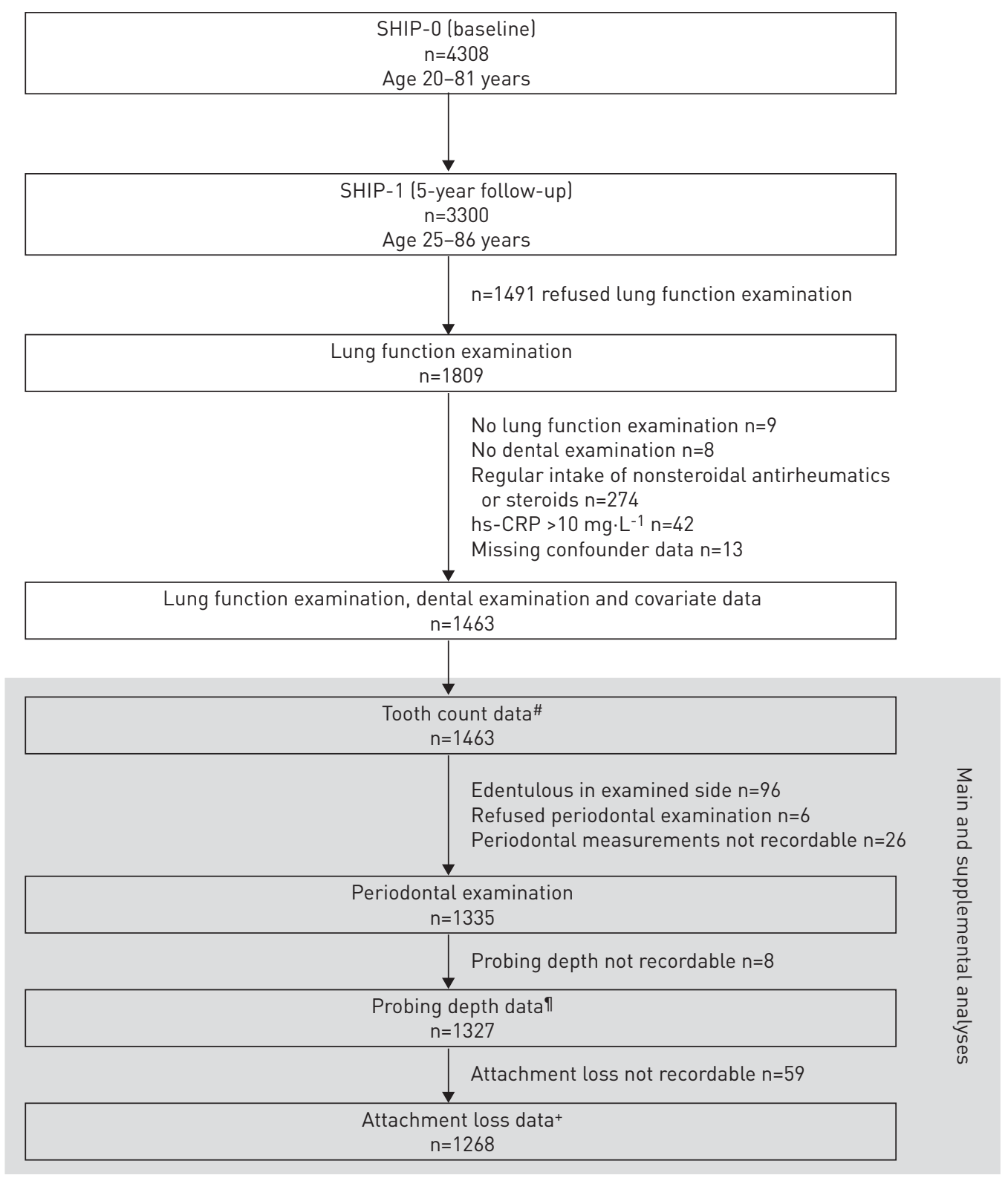

FIGURE 1 Flow chart describing the derivation of the study sample. SHIP: Study of Health in Pomerania; hs-CRP: highsensitivity C-reactive protein. ${ }^{\#}$ : see online supplementary table S1; ${ }^{\circ}$ : see online supplementary table S2; ${ }^{+}$: see tables $1-3$.

\section{Periodontal variables}

Periodontal disease was assessed by probing depth and CAL. Measurements were assessed at mesiobuccal, midbuccal, distobuccal and midpalatinal/midlingual tooth sites for all teeth except third molars, according to the half-mouth method; that is the first and fourth quadrants (right side) or the second and third quadrants (left side) were examined, alternating in each subject. A manual periodontal probe was used (PCP2; Hu-Friedy, Chicago, IL, USA). Probing depth (severity of current disease) equals the distance from the free gingival margin to the bottom of the periodontal pocket. CAL (severity of cumulative disease) equals the distance from the cemento-enamel junction to the pocket base. CAL was not recorded when cemento-enamel junction determination was indistinct (e.g. wedge-shaped defects, fillings and crown margins). Measurements were rounded to the closest millimetre. Mean CAL/probing depth over all measurement sites and the percentage of sites with $\mathrm{CAL} /$ probing depth $\geqslant 4 \mathrm{~mm}$ was calculated. The number of missing teeth excluding third molars was determined.

Dental examinations were conducted by calibrated and licenced dentists. Biannual calibration exercises on subjects not connected to the study yielded intraclass correlations of $0.70-0.89$ per examiner and an interrater correlation of 0.90 for attachment level measurements. 


\section{Lung function variables}

Details of lung function examinations that have been used in this study are given elsewhere [20]. In brief, lung function examinations were performed using a body plethysmograph equipped with a pneumotachograph (MasterScreen Body/Diff; Jaeger, Hoechberg, Germany), meeting the American Thoracic Society criteria [29] and recommendations of the European Respiratory Society [30, 31].

For the purpose of the study the following lung function variables were assessed: airway resistance (Rtot), inspiratory vital capacity, functional residual capacity derived by body plethysmography (FRCpleth), residual volume (RV) and total lung capacity (TLC). Forced vital capacity (FVC), forced expiratory volume in $1 \mathrm{~s}$ (FEV1) and maximal expiratory flow at $25 \%$ of FVC (MEF25\%) were determined by spirometry. FEV1/FVC (\%) and RV/TLC (\%) were calculated. All subjects were advised not to smoke tobacco and not to use any inhaler within $1 \mathrm{~h}$ prior to the test.

After a break of $\geqslant 3 \mathrm{~min}$, alveolar volume $(V \mathrm{~A})$ using helium dilution [31] and TLCO corrected for current haemoglobin and VA (TLCOc-VA) were measured by a single breath manoeuvre [32].

\section{Pre-exercise diagnostics}

Sociodemographic and medical variables were assessed by computer-assisted personal interviews. Information on socioeconomic status and behavioural characteristics were retrieved from the computerguided interview. School education level was categorised based on the East German three-level school system $(<10,10$ or $>10$ years). High physical activity was classified as $\geqslant 2-3 \mathrm{~h}$ per week. To precisely assess the participant's tobacco smoking, information was combined: smoking status (never/former/current), daily versus less than daily smoking, number of cigarettes smoked per day and, for former smokers, time since quitting smoking. Accordingly, participants were categorised into never-smokers, current less than daily smokers, former smokers who quit $\leqslant 10,>10$ and $\leqslant 20$ or $>20$ years ago, and current daily smokers. The number of pack-years was calculated as packs $\cdot$ day $^{-1} \times$ years of smoking. Never-smokers were those who answered "No" to the question whether they smoke or had ever smoked every day.

Body height and weight were determined using calibrated scales, and body mass index (BMI) was measured in $\mathrm{kg} \cdot \mathrm{m}^{-2}$. Waist circumference was measured to the nearest $0.1 \mathrm{~cm}$ of the narrowest part of the waist midway between the lower rib margin and the iliac crest in the horizontal plane in a standing position and with light clothing.

Previous history of diseases was based on self-reported physician's diagnosis. Use of medication influencing lung function was recorded according to the Anatomical Therapeutic Chemical (ATC) code [33]. The following drugs were considered: steroids (ATC A11ED, A14A, D07, G01B, H02, J01XC, M01BA, N02CB, R01AD, S01BA, S01BB, S01BC, S01BX, S01CA, S01CB, S02B, S02C, S03B and S03C) and nonsteroidal antirheumatics (ATC M01). Diabetes mellitus was defined as self-reported physician's diagnosis or intake of antidiabetic medication (ATC A10) in SHIP-0 or as self-reported physician's diagnosis or intake of antidiabetic medication (ATC A10) between baseline and follow-up (SHIP-1). Asthma and COPD were defined using self-reported physician's diagnosis.

\section{Blood sampling}

For laboratory examinations, nonfasting blood samples were obtained from the cubital vein in supine position. hs-CRP (Dade Behring, Eschborn, Germany), plasma fibrinogen according to the Clauss method (Electra 1600 analyser; Instrumentation Laboratory, Barcelona, Spain) and leukocyte counts (Coulter MaxM; Coulter Electronics, Miami, FL, USA) were determined.

To adjust diffusing capacity for the potential influence of blood haemoglobin, haemoglobin concentrations were determined on the same day, immediately before lung function measurements. Hence, a sample of capillary blood was taken from the earlobe and immediately transferred to the blood gas and haemoglobin analyser (Radiometer ABL 510; Radiometer, Copenhagen, Denmark).

\section{Statistical analyses}

Continuous data were expressed as median (interquartile range). Categorical data were presented as $\mathrm{n}(\%)$. Subjects were classified as having a low $(\leqslant)$ or a high $(>)$ mean CAL value compared to the age- and sexspecific median for mean CAL. Differences between subjects with low and high mean CAL were tested using Mann-Whitney U-tests (continuous data) or Chi-squared tests (categorical data).

Linear regression models were used to assess the association between periodontal and pulmonary variables. First-degree fractional polynomials were applied to explore and graph nonlinear associations [34]. To evaluate goodness of fit, deviances were compared using Chi-squared distributions with corresponding 
degrees of freedom [34]. If none of the fractional polynomial models fitted the data significantly better than the linear model, linear regression was applied.

Starting with the exposure effect estimate for mean CAL from the model including age, sex and time between core and pulmonary examination, confounders making the most difference in the estimate were added one by one (forward selection) [35]. A change in estimate of $>5 \%$ was considered important. Final models were adjusted for age, sex, time between core and pulmonary examination, diabetic status, waist circumference, presence of asthma, physical activity, smoking status, school education and height (as ageand sex-specific quintiles). Presence of COPD did not change estimates for mean CAL by $>5 \%$.

Next, to assess the possibility that systemic inflammation might link periodontal disease with lung function, fibrinogen and hs-CRP (M6) were additionally included in the full model. Relative change in estimate for mean CAL (exposure) was estimated to assess a putative intermediate position of systemic inflammation.

In sensitivity analyses, models were adjusted for either height and weight or BMI. Furthermore, subjects were restricted to never-smokers and periodontal variables were substituted by the percentage of surfaces with CAL or probing depth $\geqslant 4 \mathrm{~mm}$.

As recommended for epidemiological studies with missing data, multiple imputations of missing lung function data via chained equations were performed to reduce potential bias due to missing values in complete case analyses [36]. Using the STATA procedure interactive collaboration and evaluation with 20 runs [37], parameters of lung function were predicted by age, height, waist circumference, congestive heart failure (self-reported physician's diagnosis, echocardiographic evidence of left ventricular or valvular dysfunction or in accordance with the Rotterdam Study definition of heart failure [38]), systolic blood pressure, diabetes, evidence of coronary artery disease (myocardial infarction, signs of ischaemia in ECG), related medications (chronic use of nonsteroidal antirheumatics and steroid intake) and smoking packyears in strata of sex and smoking status [39]. Afterwards regression analyses were repeated using the imputed data.

Statistical significance was considered at a two-sided $\mathrm{p}<0.05$. Statistical analyses were performed using STATA/SE 12.0 (StataCorp, College Station, TX, USA).

\section{Results}

Subjects with high mean CAL were less often well educated, were more often current smokers, and had higher levels of systemic inflammation compared with subjects with low mean CAL (table 1). In addition, they had a deteriorated lung function $(\mathrm{p}<0.05)$.

\section{Association between mean CAL and pulmonary variables}

In the overall sample (table 2), significant associations of mean CAL with dynamic lung volumes (FVC (fig. 2a) and FEV1 (fig. 2b)), static lung volumes (FRCpleth) and airflow limitation (FEV1/FVC ratio, $\mathrm{MEF} 25 \%$, Rtot and RV/TLC ratio (fig. 2c)) were seen after full adjustment for potential confounders. Restricted to never-smokers, mean CAL was significantly $(\mathrm{p}<0.05)$ associated with dynamic lung volumes (FVC and FEV1) and with RV/TLC ratio (fig. 2). Data imputation confirmed associations with dynamic lung volumes (FVC and FEV1), static lung volumes (FRCpleth) and airflow limitation (FEV1/FVC ratio, $\mathrm{MEF} 25 \%$ and RV/TLC ratio).

To assess the strength of confounding by smoking, height and education more closely, stepwise adjusted models were evaluated (table 3). After adjustment for age, sex, time between core and pulmonary examination, diabetes, waist circumference, physical activity and asthma (M1), mean CAL was significantly related to all pulmonary variables $(\mathrm{p}<0.05)$, except TLC. Adjustment for smoking (M2) reduced coefficients for mean CAL by $>5 \%$ for all variables except Rtot. Further adjustment for body height (M3) led to a more pronounced change in coefficients of mean CAL in relation to FVC, FEV1, MEF25\%, FRCpleth and TLC compared with an adjustment for education (M4). In the full model (M5), mean CAL was significantly associated with FVC, FEV1, FEV1/FVC ratio, MEF25\%, Rtot, FRCpleth and RV/TLC ratio $(\mathrm{p}<0.05)$. Higher levels of mean CAL were related to lower levels of FVC (fig. 2a) and FEV1 (fig. 2b) and higher levels of RV/TLC ratio (fig. 2c). In addition, fibrinogen and hs-CRP were considered as intermediates linking periodontal disease with lung function. For those pulmonary variables showing significant associations with mean CAL in the full model (M5), inclusion of fibrinogen and hs-CRP (M6) did not change coefficients of mean CAL.

\section{Sensitivity analyses}

Considering mean probing depth as periodontal exposure (online supplementary table S2), relevant associations with FEV1, FEV1/FVC ratio, FRCpleth and RV/TLC ratio were seen in the overall sample 
TABLE 1 Characteristics of the study population stratified by mean clinical attachment loss (CAL)

Mean CAL

p-value

\begin{tabular}{c}
\hline Low \\
640 \\
$18(0-71)$ \\
$4001(3367-4744)$ \\
$3417(2882-4123)$ \\
$85.8(82.2-88.9)$ \\
$1.60(1.18-2.08)$ \\
$0.21(0.17-0.27)$ \\
$3.54(3.02-4.15)$ \\
$6300(5475-7207)$ \\
$34.4(28.5-40.2)$ \\
$1.452(1.316-1.605)$ \\
$0.94(0.37-1.75)$ \\
$2.0(0-10.0)$ \\
$1.89(1.71-2.09)$ \\
$2.3(0-6.8)$ \\
$24(22-27)$ \\
$1.0(0.6-2.2)$ \\
$2.9(2.5-3.4)$ \\
$6.3(5.2-7.2)$
\end{tabular}

High

\section{Subjects $n$ \\ Lung function variables}

Time between core and pulmonary examination days

Dynamic lung volumes

FVC $\mathrm{mL}$

FEV1 $\mathrm{mL}$

Obstruction/airflow limitation

FEV $1 / F V C \%$

MEF $25 \% L \cdot S^{-1}$

Rtot $\mathrm{kPa} \cdot \mathrm{S}^{-1} \cdot \mathrm{L}^{-1}$

Static lung volumes

FRCpleth $\mathrm{mL}$

TLC $\mathrm{mL}$

RV/TLC \%

Diffusion

TLCOC-VA mmol $\cdot \mathrm{kPa}^{-1} \cdot \mathrm{min}^{-1} \cdot \mathrm{L}^{-1}$

\section{Periodontal variables}

Mean CAL mm

Percentage of sites with CAL $\geqslant 4 \mathrm{~mm} \%$

Mean PD mm

Percentage of sites with PD $\geqslant 4 \mathrm{~mm} \%$

Tooth count

\section{Laboratory variables}

High-sensitivity CRP $\mathrm{mg} \cdot \mathrm{L}^{-1}$

Fibrinogen (Clauss) $\mathrm{g} \cdot \mathrm{L}^{-1}$

Leukocyte count $\mathrm{Gpt} \cdot \mathrm{L}^{-1}$

$6.3(5.2-7.2)$

Subject characteristics

Male

50.9

Age at core examination years

School education

$<10$ years

10 years

$>10$ years

Smoking status

Never-smoker

Current less-than-daily smoker

Former smoker quit $\leqslant 10$ years ago

Former smoker quit $>10-\leqslant 20$ years ago

Former smoker quit $>20$ years ago

Current daily smoker

Pack-years

Diabetes mellitus

High physical activity ${ }^{+}$

Body mass index $\mathrm{kg} \cdot \mathrm{m}^{-2}$

Height $\mathrm{cm}$

Weight kg

Waist circumference $\mathrm{cm}$

$50.1(39.9-60.3)$

Medical diseases

COPD

Asthma

Coronary artery disease ${ }^{\S}$

18.8
54.8
26.4
49.7
4.1
10.0
8.7
12.8
14.7
$0(0-6.9)$
4.5
22.8
$26.8(24.1-29.8)$
$171(164-178)$
$79.4(69.1-89.8)$
$91(82-100)$

1.3
3.8
4.4

628

$19(0-82)$

0.11

3882 (3269-4668) $\quad 0.06$

$3298(2748-3960) \quad 0.01$

$85.2(81.5-88.8) \quad 0.056$

$1.50(1.09-1.97) \quad 0.008$

$\begin{array}{ll}0.22(0.17-0.28) & 0.17\end{array}$

$3.55(2.99-4.17) \quad 0.75$

$6317(5398-7225) \quad 0.82$

$35.6(29.7-41.9) \quad 0.005$

$1.406(1.267-1.596) \quad 0.009$

$3.03(1.96-4.25)<0.001$

$35.6(7.7-67.3)<0.001$

$2.46(2.05-2.97) \quad<0.001$

$16.0(4.2-32.7)<0.001$

$22(15-26) \quad<0.001$

$1.3(0.6-2.7) \quad 0.003$

$3.0(2.6-3.6) \quad<0.001$

$6.5(5.5-7.8)<0.001$

51.1

0.95

$50.1(39.8-60.2)$

0.99

26.8

55.4

17.8

$<0.001$

39.3

2.2

12.1

5.6

12.0

28.8

$3.0(0-15.1)$

6.7

20.1

$27.2(24.5-30.8)$

$170(164-177)$

79.4 (69.1-91.8)

$92(82-102)$

$<0.001$

$<0.001$

0.10

0.23

0.03

0.11

0.44

0.27

0.46

0.002

0.46

Data are given as median (interquartile range) or \%, unless otherwise stated. $n=1268$. FVC: forced vital capacity; FEV1: forced expiratory volume in $1 \mathrm{~s}$; MEF25\%: maximal expiratory flow at $25 \%$ of FVC; Rtot: total airway resistance; FRCpleth: functional residual capacity derived by body plethysmography; TLC: total lung capacity; RV: residual volume; TLCOC-VA: diffusing capacity of the lung for carbon monoxide (single breath), corrected for haemoglobin level and alveolar volume (assessed by helium dilution); PD: probing depth; CRP: C-reactive protein; COPD: chronic obstructive pulmonary disease (subject-reported physician's diagnosis). " : classified as equal to or less than (low) or greater than (high) the ageand sex-specific median for mean $\mathrm{CAL}^{\top}{ }^{\uparrow}$ : $\mathrm{p}$-values calculated by Mann-Whitney U-test for continuous data and Chi-squared test for categorical data; ${ }^{+}$: defined as sporting activity of $\geqslant 2-3 \mathrm{~h}$ per week; ${ }^{\S}$ : defined as myocardial infarction or signs of ischaemia in ECG. 
TABLE 2 Linear regression evaluating the associations between mean clinical attachment loss (CAL) (exposure) and pulmonary variables (outcomes) using fractional polynomials (FPs) in the overall sample, in never-smokers and using imputed data

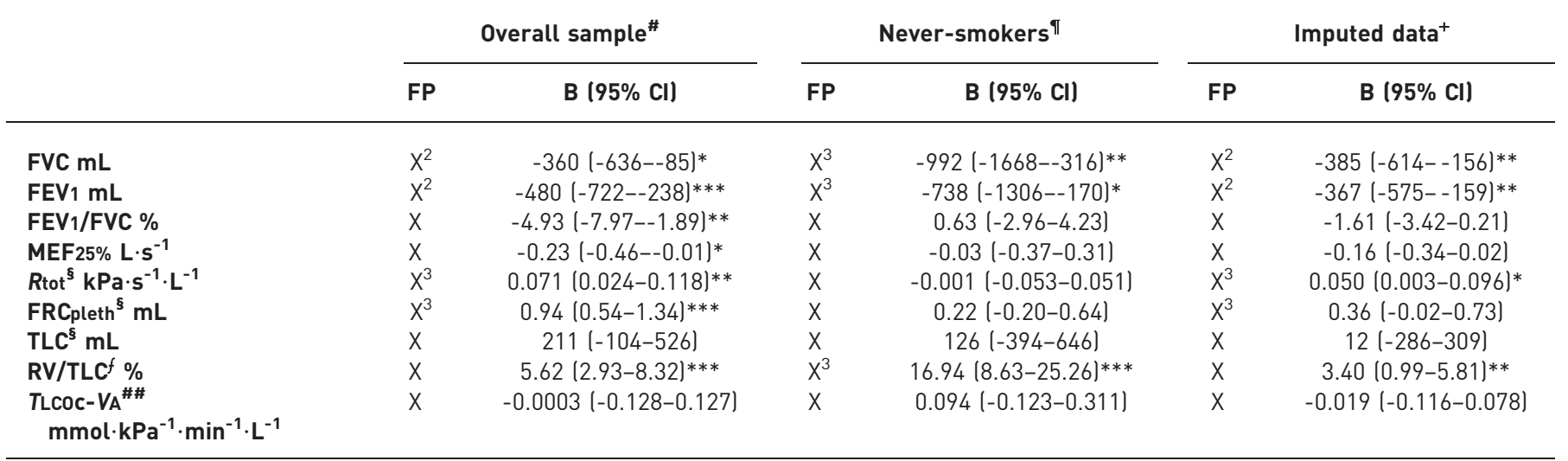

Linear regression using FPs for mean CAL adjusted for age, sex, time between core and pulmonary examination, diabetes, waist circumference, physical activity ( $\geqslant 2-3 \mathrm{~h}$ per week), asthma, chronic obstructive pulmonary disease, smoking status (six categories), height (age- and sex-specific quintiles) and school education. B: regression coefficient; FVC: forced vital capacity; FEV1: forced expiratory volume in 1 s; MEF25\%: maximal expiratory flow at 25\% of FVC; Rtot: total airway resistance; FRCpleth: functional residual capacity derived by body plethysmography; TLC: total lung capacity; RV: residual volume; TLCOc-VA: diffusing capacity of the lung for carbon dioxide (single breath), corrected for haemoglobin level and alveolar volume lassessed by helium dilution); X: mean CAL/10. ${ }^{\#}: \mathrm{n}=1268 ;{ }^{\circledR}: \mathrm{n}=565 ;{ }^{+}: \mathrm{n}=2577 ;^{\S}$ : one additional missing value; ${ }^{f}$ : three additional missing values; ${ }^{\# \#:} 12$ additional missing values in the overall sample model and seven additional missing values in never-smokers. ${ }^{*}: p<0.05$; ${ }^{* *}: \mathrm{p}<0.01 ;{ }^{* * *}: \mathrm{p}<0.001$.

$(\mathrm{p}<0.05)$. Restricting subjects to never-smokers, the association with FVC missed statistical significance $(\mathrm{p}=0.063)$. Data imputation confirmed these results. Associations were highly confounded by smoking and body height (online supplementary table S3).

Considering the number of missing teeth as the exposure (online supplementary table S1), significant associations with FVC, FEV1, FEV1/FVC ratio, Rtot, FRCpleth and RV/TLC ratio were found $(\mathrm{p}<0.05)$. Restricting analyses to never-smokers, number of missing teeth was significantly associated with FVC and TLCOc-VA $(\mathrm{p}<0.05)$. Evaluation of imputed data confirmed the associations with FVC, FEV1, FEV1/FVC, Rtot, and RV/TLC ratio $(\mathrm{p}<0.05)$.

Results were confirmed by exchanging the exposure variable with the percentage of sites with CAL/probing depth $\geqslant 4 \mathrm{~mm}$. Substituting height and waist circumference by either body height and weight or BMI did not change the results substantially.

\section{Discussion}

In this study, periodontal disease was independently associated with reduced dynamic and static lung volumes and with airflow limitation, but not with diffusing capacity. These associations, however, were
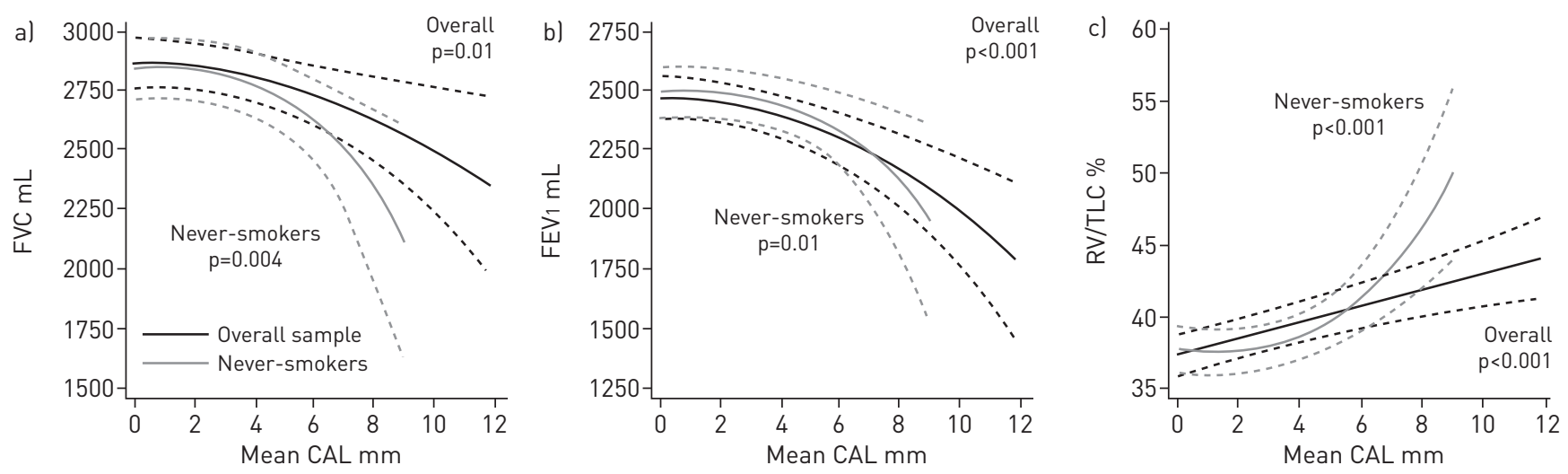

FIGURE 2 Associations between mean clinical attachment loss (CAL) and a) forced vital capacity (FVC), b) forced expiratory volume in $1 \mathrm{~s}$ (FEV1) and c)

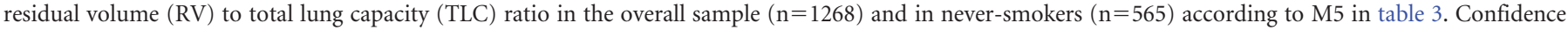
limits (dashed lines) and p-values from fully adjusted regression analyses are given. 


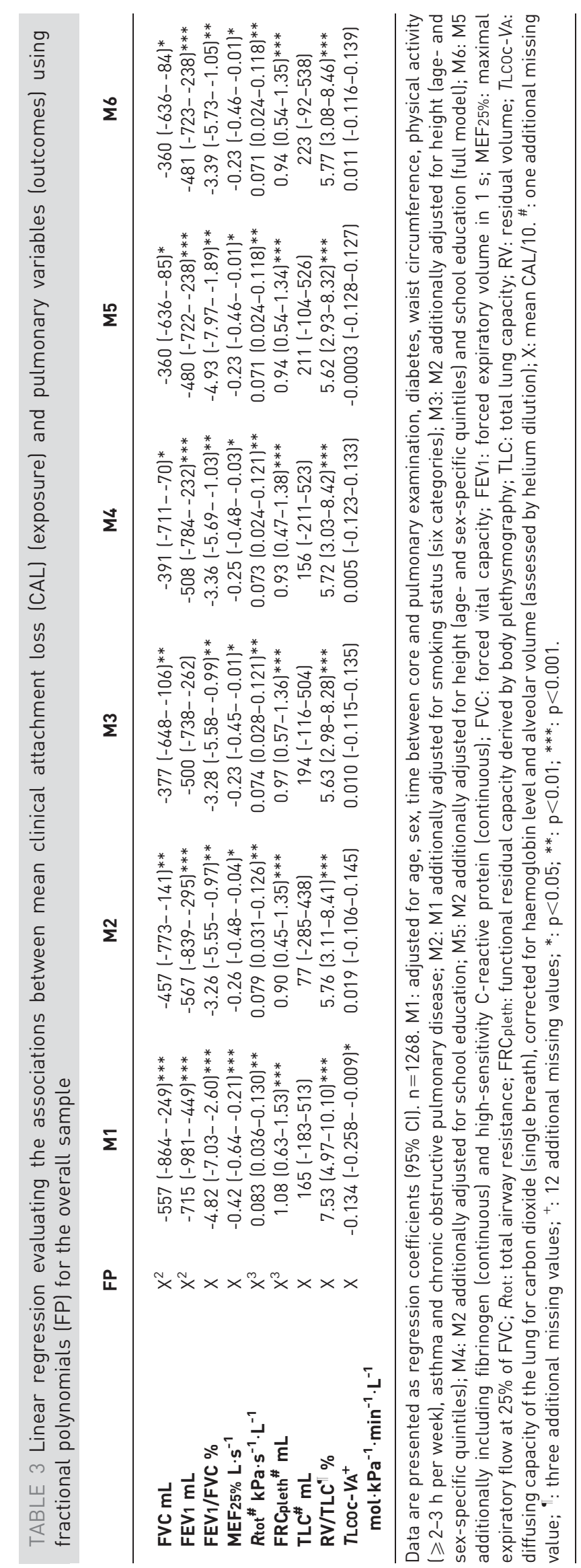


heavily confounded by smoking and body height. Systemic inflammation, as assessed by serum fibrinogen levels and hs-CRP, seemed to mediate associations between periodontal disease and lung function only to a minor degree. In never-smokers, a deteriorated periodontal status was associated with lower lung volumes (FVC and FEV1) and higher RV/TLC ratios. Our findings are consistent with previous evidence about associations between periodontal disease and airflow limitation in predefined populations with COPD [4-10]. Importantly, our study offers more accurate definitions of airflow limitation, providing a comprehensive evaluation of lung function.

Dental plaque induces gingivitis and is the primary aetiological factor for the progression of periodontal diseases [40]. Thus, the effective removal of dental plaque is essential for the prevention of periodontal disease. In SHIP, the percentage of positive plaque sites was associated with lower lung volumes (FVC and FEV1; $\mathrm{p}=0.02)$ and higher RV/TLC ratios $(\mathrm{p}=0.004$; data not shown). Results are in agreement with other studies having reported relationships between plaque and/or oral hygiene with pneumonia [15], ventilatorassociated pneumonia [41, 42] and COPD [12]. Oral hygiene may be efficient in reducing home-associated pneumonia $[15,43]$. Further, several meta-analyses $[41,42,44]$ support the use of oral antiseptics to reduce risk of ventilator-associated pneumonia. Because oral colonisation by oral or respiratory pathogens was not assessed in this study, associations between pathogen burden and lung function were not feasible. However, these studies considerably complement the scientific evidence for an association between periodontitis and lung diseases.

Besides hygiene issues in pneumonia and COPD, associations of oral microorganisms and pulmonary infections due to aspiration have been investigated [45]. Usually, aspiration pneumonia is related to lessvirulent bacteria, streptococci and anaerobes that reside in the upper airways or the gastrointestinal tract [45].

Smoking mainly confounds the association between periodontitis and lung function [5]. Smoking is involved in the pathogenesis and progression of periodontal diseases [46]. The peripheral vasoconstrictive effect of tobacco smoke and nicotine reduces the delivery of oxygen and nutrients to gingival tissue [47] and alters gingival inflammatory response [46]. Smoking also affects respiratory defences, promotes chronic lung diseases in respiratory infections, damages the airway epithelium and has effects on lung clearance by suppressing cough and the protective waving action of cilia in the airways [48].

In this study, inclusion of smoking status strongly attenuated coefficients in our models that evaluated lung volumes, airway limitation and diffusion capacity. Because we considered smoking status and number of pack-years for adjustment, residual confounding by smoking was reduced considerably. However, residual confounding by the number of cigarettes smoked appears to be an aspect consistently observed in the current literature, where associations between periodontitis and lung function were only reported among current smokers $[5,10]$. To avoid residual confounding by active smoking experience, analyses were also restricted to never-smokers. Restricting analyses to never-smokers halved the number of pulmonary variables associated with mean CAL. This aspect underlines the importance of major confounding by smoking and number of cigarettes smoked on the relationship between periodontal diseases and lung function.

It has been previously described that the socioeconomic status is associated with lung function independently of smoking [49]. In line with this, in our study adjustment for education attenuated associations between periodontal disease and airflow limitation, quantified by FEV1/FVC ratio, Rtot, RV/ TLC ratio, and diffusion, quantified by TLCOC-VA, more strongly compared with body height in our study. However, having only adjusted for education, residual confounding by other socioeconomic factors cannot be ruled out.

In this study, adjustment for body height changed coefficients of mean CAL for static and dynamic lung volumes and $\mathrm{MEF} 25 \%$ by $>10 \%$. This leads to the question of how body height might be related to both lung volume and periodontitis. Body height reflects intrauterine influences and birthweight, as well as weight and environmental factors in early childhood (e.g. nutrition, illness and psychosocial stress), which in consequence constrain the growth of the airways and may be reflected in reduced lung volumes. These constraints occurring in the fetus and early childhood may also hamper inflammatory responses to infections [50-52]. Meisel et al. [53] showed that body height was associated with periodontitis and concluded that individuals born with a high susceptibility to infectious and inflammatory diseases in childhood may present impaired growth in length. Upon reaching adulthood, growth comes to a halt, but the individual remains susceptible to inflammatory sequelae. Thus, smaller persons tend to develop more severe periodontitis.

A number of potential mechanisms have been proposed to explain the biological plausibility for an association between periodontal disease and lung function [54,55]. The oral cavity provides an optimal environment for bacterial growth because of its humidity and temperature. $1 \mathrm{~mm}^{3}$ of plaque contains $>10^{6}$ bacteria with 300 different anaerobic and facultative anaerobic species [56]. The most common route by 
which the oral cavity may influence pulmonary function is the aspiration of saliva harbouring oral bacteria into the lung [24]. Aspiration of small amounts of saliva during sleep is quite common, even in healthy subjects [57]. Subjects with severe periodontal disease harbour elevated levels of periodontal pathogens in saliva compared with healthy subjects [58]. The amount of pathogens reflects pathogen burden in periodontal pockets [58]. When aspirated, oral pathogens cause local damage to the small airways [57].

Furthermore, oral bacteria stimulate the inflamed periodontal tissues to release cytokines via the sulcus fluid into the saliva. In subjects with periodontal disease salivary biomarker load is higher than in healthy subjects [58]. Aspirated cytokines may promote the adhesion and growth of respiratory pathogens in the lower airways [54]. Subsequently, the respiratory epithelium may release cytokines and attract neutrophils, which in turn infiltrate airway parenchyma and release proteolytic enzymes and toxic oxygen radicals which damage the epithelium $[59,60]$. As a consequence, the resultant inflamed mucosal epithelium may be particularly prone to infection.

Low-grade systemic inflammation, specifically addressed in this study, might provide a further plausible link. Elevated levels of cytokines and higher levels of circulating inflammatory markers, such as CRP and fibrinogen, have not only been associated with periodontal diseases [16], but also with reduced lung function [17-20]. However, restricting models to those showing significant associations with mean CAL after full adjustment, inclusion of fibrinogen and hs-CRP did not reduce coefficients for mean CAL by $>5 \%$. Thus, systemic inflammation might only be of minor importance when considering the link between periodontal diseases and lung volume. Nevertheless, results are in agreement with others showing that hsCRP and fibrinogen were associated with TLC [17-20].

There are several strengths of our analysis, the major one being the population-based approach including a general German adult population sample with a broad age range (25-85 years). Second, various clinical periodontal definitions were used, covering both current (probing depth) and cumulative disease extent (attachment loss and tooth count); all definitions presented a consistent impression of the effect of periodontitis on lung function. Dental examiners had been calibrated twice a year. Third, a highly standardised method in obtaining comprehensive lung function parameters using standard spirometry, body plethysmography, helium dilution for measurement of alveolar volume and diffusing capacity measurement for carbon monoxide was used.

Three limitations of our study merit comment. One of these is its cross-sectional design, known to preclude the establishment of a definite cause and effect relationship. Second, selection bias due to nonparticipation in pulmonary function tests may have occurred; younger and healthier subjects volunteered and no subjects with advanced COPD were identified among the study participants. To counter this, multiple imputations of missing lung function data via chained equations were performed to reduce potential bias due to missing values in complete case analyses [36]. After imputation, associations between periodontal disease and lung function were confirmed. Third, since periodontal measurements were taken half-mouth, extent of periodontal disease might be underestimated, and observed associations with lung function might be biased towards the null [61]. However, use of mean values of probing depth and CAL limits underestimation and bias of risk estimates.

\section{Conclusion}

In conclusion, periodontal disease was significantly associated with reduced lung volumes, hyperinflation and airflow limitation in this general population sample independently of potential confounders. Smoking and height highly confounded the association between periodontitis and lung function. Systemic inflammation did not provide a mechanism linking both diseases. In future, large cohort studies with long observation periods evaluating comprehensive lung function parameters are needed to validate the observed associations and to assess causality more closely. In this context, a comprehensive consideration of confounding bias is strongly suggested and analyses restricted to never-smokers are encouraged. Further investigations on the association between oral pathogen burden and lung function would complement results from clinical trials. Furthermore randomised clinical trials are needed to investigate whether prevention or treatment of periodontitis might have a beneficial impact on lung function.

\section{Acknowledgements}

The contributions to data collection made by all contributors are gratefully acknowledged.

\section{References}

1 Demmer RT, Papapanou PN. Epidemiologic patterns of chronic and aggressive periodontitis. Periodontol 2000 2010; 53: 28-44.

2 Holtfreter B, Schwahn C, Biffar R, et al. Epidemiology of periodontal diseases in the Study of Health in Pomerania. J Clin Periodontol 2009; 36: 114-123. 
Pihlstrom BL, Michalowicz BS, Johnson NW. Periodontal diseases. Lancet 2005; 366: 1809-1820.

Deo V, Bhongade ML, Ansari S, et al. Periodontitis as a potential risk factor for chronic obstructive pulmonary disease: a retrospective study. Indian J Dent Res 2009; 20: 466-470.

5 Hyman JJ, Reid BC. Cigarette smoking, periodontal disease, and chronic obstructive pulmonary disease. J Periodontol 2004; 75: 9-15.

6 Katancik JA, Kritchevsky S, Weyant RJ, et al. Periodontitis and airway obstruction. J Periodontol 2005; 76: Suppl. 11, 2161-2167.

7 Scannapieco FA, Ho AW. Potential associations between chronic respiratory disease and periodontal disease: analysis of National Health and Nutrition Examination Survey III. J Periodontol 2001; 72: 50-56.

8 Wang Z, Zhou X, Zhang J, et al. Periodontal health, oral health behaviours, and chronic obstructive pulmonary disease. J Clin Periodontol 2009; 36: 750-755.

9 Hayes C, Sparrow D, Cohen M, et al. The association between alveolar bone loss and pulmonary function: the VA Dental Longitudinal Study. Ann Periodontol 1998; 3: 257-261.

10 Garcia RI, Nunn ME, Vokonas PS. Epidemiologic associations between periodontal disease and chronic obstructive pulmonary disease. Ann Periodontol 2001; 6: 71-77.

11 Leuckfeld I, Obregon-Whittle MV, Lund MB, et al. Severe chronic obstructive pulmonary disease: association with marginal bone loss in periodontitis. Respir Med 2008; 102: 488-494.

12 Si Y, Fan H, Song Y, et al. Association between periodontitis and chronic obstructive pulmonary disease in a Chinese population. J Periodontol 2012; 83: 1288-1296.

13 Sharma N, Shamsuddin H. Association between respiratory disease in hospitalized patients and periodontal disease: a cross-sectional study. J Periodontol 2011; 82: 1155-1160.

14 Hämäläinen P, Suominen H, Keskinen M, et al. Oral health and reduction in respiratory capacity in a cohort of community-dwelling elderly people: a population-based 5-year follow-up study. Gerodontology 2004; 21: $209-215$.

15 Yoneyama T, Yoshida M, Ohrui T, et al. Oral care reduces pneumonia in older patients in nursing homes. $J$ Am Geriatr Soc 2002; 50: 430-433.

16 Yoshii S, Tsuboi S, Morita I, et al. Temporal association of elevated C-reactive protein and periodontal disease in men. J Periodontol 2009; 80: 734-739.

17 Thyagarajan B, Jacobs DR, Apostol GG, et al. Plasma fibrinogen and lung function: the CARDIA Study. Int $J$ Epidemiol 2006; 35: 1001-1008.

18 Shaaban R, Kony S, Driss F, et al. Change in C-reactive protein levels and FEV1 decline: a longitudinal populationbased study. Respir Med 2006; 100: 2112-2120.

19 Rasmussen F, Mikkelsen D, Hancox RJ, et al. High-sensitive C-reactive protein is associated with reduced lung function in young adults. Eur Respir J 2009; 33: 382-388.

20 Gläser S, Ittermann $\mathrm{T}$, Koch B, et al. Airflow limitation, lung volumes and systemic inflammation in a general population. Eur Respir J 2012; 39: 29-37.

21 Tamimi A, Serdarevic D, Hanania NA. The effects of cigarette smoke on airway inflammation in asthma and COPD: therapeutic implications. Respir Med 2012; 106: 319-328.

Zammit C, Liddicoat H, Moonsie I, et al. Obesity and respiratory diseases. Int J Gen Med 2010; 3: $335-343$. Guerra S. Asthma and chronic obstructive pulmonary disease. Curr Opin Allergy Clin Immunol 2009; 9: 409-416. Scannapieco FA, Stewart EM, Mylotte JM. Colonization of dental plaque by respiratory pathogens in medical intensive care patients. Crit Care Med 1992; 20: 740-745.

25 John U, Greiner B, Hensel E, et al. Study of Health In Pomerania (SHIP): a health examination survey in an east German region: objectives and design. Soz Praventivmed 2001; 46: 186-194.

26 Völzke H, Alte D, Schmidt CO, et al. Cohort profile: the study of health in Pomerania. Int J Epidemiol 2011; 40: 294-307.

27 Haring R, Alte D, Völzke H, et al. Extended recruitment efforts minimize attrition but not necessarily bias. J Clin Epidemiol 2009; 62: 252-260.

28 Spitzer C, Barnow S, Völzke H, et al. Association of posttraumatic stress disorder with low-grade elevation of C-reactive protein: evidence from the general population. J Psychiatr Res 2010; 44: 15-21.

29 Nelson SB, Gardner RM, Crapo RO, et al. Performance evaluation of contemporary spirometers. Chest 1990; 97: $288-297$.

30 Miller MR, Hankinson J, Brusasco V, et al. Standardisation of spirometry. Eur Respir J 2005; 26: 319-338.

31 Wanger J, Clausen JL, Coates A, et al. Standardisation of the measurement of lung volumes. Eur Respir J 2005; 26: 511-522.

32 Macintyre N, Crapo RO, Viegi G, et al. Standardisation of the single-breath determination of carbon monoxide uptake in the lung. Eur Respir J 2005; 26: 720-735.

33 WHO Collaborating Centre for Drug Statistics Methodology. Guidelines for ATC classification and DDD assignment. Oslo, WHO Collaborating Centre for Drug Statistics Methodology, 2008.

34 Royston P, Ambler G, Sauerbrei W. The use of fractional polynomials to model continuous risk variables in epidemiology. Int J Epidemiol 1999; 28: 964-974.

35 Greenland S, Rothman KJ. Introduction to stratified analysis. In: Greenland S, Rothman KJ, Lash TL, eds. Modern Epidemiology. 3rd Edn. Philadelphia, Lippincott Williams \& Wilkins, 2008; pp. 258-282.

36 van Buuren S, Boshuizen HC, Knook DL. Multiple imputation of missing blood pressure covariates in survival analysis. Stat Med 1999; 18: 681-694.

37 Sterne JA, White IR, Carlin JB, et al. Multiple imputation for missing data in epidemiological and clinical research: potential and pitfalls. BMJ 2009; 338: b2393.

38 Bleumink GS, Knetsch AM, Sturkenboom MC, et al. Quantifying the heart failure epidemic: prevalence, incidence rate, lifetime risk and prognosis of heart failure. The Rotterdam Study. Eur Heart J 2004; 25: 1614-1619.

39 Arnold AM, Kronmal RA. Multiple imputation of baseline data in the cardiovascular health study. Am J Epidemiol 2003; 157: 74-84.

40 Marsh PD. Dental plaque as a biofilm and a microbial community - implications for health and disease. BMC Oral Health 2006; 6: Suppl. 1, S14.

41 Labeau SO, Van de Vyver K, Brusselaers N, et al. Prevention of ventilator-associated pneumonia with oral antiseptics: a systematic review and meta-analysis. Lancet Infect Dis 2011; 11: 845-854. 

adults: systematic review and meta-analysis. BMJ 2007; 334: 889.

43 Bassim CW, Gibson G, Ward T, et al. Modification of the risk of mortality from pneumonia with oral hygiene care. J Am Geriatr Soc 2008; 56: 1601-1607.

44 Chlebicki MP, Safdar N. Topical chlorhexidine for prevention of ventilator-associated pneumonia: a meta-analysis. Crit Care Med 2007; 35: 595-602.

45 Pace CC, McCullough GH. The association between oral microorgansims and aspiration pneumonia in the institutionalized elderly: review and recommendations. Dysphagia 2010; 25: 307-322.

46 Bergström J. Tobacco smoking and chronic destructive periodontal disease. Odontology 2004; 92: 1-8.

47 Baab DA, Oberg PA. The effect of cigarette smoking on gingival blood flow in humans. J Clin Periodontol 1987; 14: 418-424.

48 Terpenning MS. The relationship between infections and chronic respiratory diseases: an overview. Ann Periodontol 2001; 6: 66-70.

49 Prescott E, Lange P, Vestbo J. Socioeconomic status, lung function and admission to hospital for COPD: results from the Copenhagen City Heart Study. Eur Respir J 1999; 13: 1109-1114.

50 Barker DJ, Godfrey KM, Fall C, et al. Relation of birth weight and childhood respiratory infection to adult lung function and death from chronic obstructive airways disease. BMJ 1991; 303: 671-675.

51 Marossy AE, Strachan DP, Rudnicka AR, et al. Childhood chest illness and the rate of decline of adult lung function between ages 35 and 45 years. Am J Respir Crit Care Med 2007; 175: 355-359.

52 Sattar N, McConnachie A, O’Reilly D, et al. Inverse association between birth weight and C-reactive protein concentrations in the MIDSPAN Family Study. Arterioscler Thromb Vasc Biol 2004; 24: 583-587.

53 Meisel P, Kohlmann T, Kocher T. Association of height with inflammation and periodontitis: the Study of Health in Pomerania. I Clin Periodontol 2007; 34: 390-396.

54 Scannapieco FA. Role of oral bacteria in respiratory infection. J Periodontol 1999; 70: 793-802.

55 Scannapieco FA, Bush RB, Paju S. Associations between periodontal disease and risk for nosocomial bacterial pneumonia and chronic obstructive pulmonary disease. A systematic review. Ann Periodontol 2003; 8: 54-69.

56 Slots J, Rams TE, Listgarten MA. Yeasts, enteric rods and pseudomonads in the subgingival flora of severe adult periodontitis. Oral Microbiol Immunol 1988; 3: 47-52.

57 Sinclair DG, Evans TW. Nosocomial pneumonia in the intensive care unit. Br J Hosp Med 1994; 51: 177-180.

58 Saygun I, Nizam N, Keskiner I, et al. Salivary infectious agents and periodontal disease status. J Periodontal Res 2011; 46: 235-239.

59 Birkedal-Hansen H. Role of cytokines and inflammatory mediators in tissue destruction. J Periodontal Res 1993; 28: 500-510.

60 Pesci A, Balbi B, Majori M, et al. Inflammatory cells and mediators in bronchial lavage of patients with chronic obstructive pulmonary disease. Eur Respir J 1998; 12: 380-386.

61 Beck JD, Pankow J, Tyroler HA, et al. Dental infections and atherosclerosis. Am Heart J 1999; 138: S528-S533. 